\title{
DISTRIBUIÇÃO DO SISTEMA RADICULAR DO MILHO EM TERRA ROXA ESTRUTURADA LATOSSÓLICA: II. COMPARAÇÃO ENTRE CULTURA IRRIGADA E FERTIRRIGADA ${ }^{1}$
}

\author{
L.H. BASSOI \\ CPTASA/EMBRAPA, C.P. 23 - CEP: 56300-000 - Petrolina,PE \\ L. FANTE JÚNIOR \\ Centro de Energia Nuclear na Agricultura/USP - C.P. 96 - CEP: 13400-970 - Piracicaba,SP \\ L.A.C. JORGE; S. CRESTANA \\ CNPDIA/EMBRAPA, C.P. 741, CEP; $13560-970$ - Säo Carlos,SP \\ K. REICHARDT \\ Departamento de Física e Meteorologia-ESALQ/USP, C.P. 9, CEP: 13418-900 - Piracicaba,SP e Centro de Energia \\ Nuclear na Agricultura/USP, C.P. 96 - CEP: 13400-970 - Piracicaba,SP
}

\begin{abstract}
RESUMO: A distribuição do sisterma radicular do milho irrigado, atê $1 \mathrm{~m}$ de profundidade, em uma terra roxa estruturada latossólica em Piracicaba, SP, foi analisada aos 126 dias após a emergência das plantas. As raízes expostas pela abertura de trincheiras longitudinalmente ass linhas de plantas foram filmadas e essas imagens digitalizadas e processadas para a contabilização de sua distribuição no perfil do solo, por meio do sistema SIARCS desenvolvido para esse fim. A densidade de raiz de milho a $0-60 \mathrm{~cm}$ foi maior para a aplicação de $\mathrm{N}$ via solo, enquanto que a $60-100 \mathrm{~cm}$ a fertirrigação proporcionou uma maior presença de raízes. Por meio de tensiômetros e da sonda de nêutrons, notou-se, respectivamente, que a maior amplitude de variação do potencial matricial da água no solo e da quantidade de água armazenada no solo ocorreu até a profundidade de $50 \mathrm{~cm}$, evidenciando a boa correspondência dessas técnicas com o método de processamento de imagens.
\end{abstract}

Descritores: distribuição radicular, milho, irrigaçãa, processamento de imagens

\section{DISTRIBUTION OF MAIZE ROOT SYSTEM IN A KANDUIDALFIC EUTRUDOX SOIL: II. COMPARISON BETWEEN IRRIGATED AND FERTIRRIGATED CROPS}

\begin{abstract}
ARSTRACT: A field experiment was carried out to evaluate the distribution of irrigated maize root system in a 1 $\mathrm{m}$ soil layer of terra roxa estruturada latossolica (Kanduidalfic Eutrudox soil) in Piracicaba, SP, Brazil (22 ${ }^{\circ} 43^{\prime} \mathrm{S}$, $47^{\circ} 5^{\prime} \mathrm{W}$, at 126 days after plant emergence. The root system exposed in trenches was filmed and the pictures processed by a system SIARCS, specially developed to analyze root system distributions. Maize root density in the 0-60 cm soil layer was higher for the case of $N$ application to the soil, whereas for the $60-100 \mathrm{~cm}$ fertirrigation promoted root development. Soil water matric potential and the water storage in the soil profile presented greater changes to the $50 \mathrm{~cm}$ depth, showing a good relation between tensiometry, neutron probe measurements and the picture processing method.
\end{abstract}

Key Words: root system distribution, com, irrigation, pictures processing method

\section{INTRODUÇÃo}

O crescimento do sistema radicular de uma cultura está condicionado a fatores químicos, físicos e biológicos do solo e o conhecimento de sua distribuição é importante para a compreensão da dinâmica da água e de nutrientes no solo. $O$ manejo de irrigação depende, entre outros, da profundidade das raízes, para que se tenha uma reposição de água na camada de solo em que se encontra a maior parte do sistema radicular ativo, de modo a minimizar as perdas por lixiviação.

\footnotetext{
${ }^{1}$ Parte da Tese de Doutorado do primeiro autor apresentada ao Centro de Energia Nuclear na Agricultua (CENA/USP).
} 
REICHARDT (1981) cita que o $\mathrm{pH}$ do solo, o teor de alumínio trocável, a densidade global, a armazenagem de água e a condutividade hidráulica influenciam o crescimento radicular e a absorção de nutrientes. Segundo DEMATTÊ (1981), o desenvolvimento radicular pode ser comprometido pela ação de algumas características adversas dos solos, tais como compactação devido ao manejo ou aos processos pedogenéticos, toxidade de elementos químicos e excesso de água. Em regiões úmidas brasileiras, como a Amazônia, parte do Cerrado, região Sudeste e Sul do Brasil, a toxidade de alumínio e a falta de alguns nutrientes, como o cálcio, magnésio e potássio, são os principais fatores que podem comprometer o desenvolvimento das raízes.

WAHAB et al. (1976) ao compararem a profundidade de enraizamento de plantas de milho, notaram que a máxima profundidade atingida pelo sistema radicular em um oxisol foi de $90-95 \mathrm{~cm}$, com $75 \%$ do total a $50 \mathrm{~cm}$, enquanto que em um ultisol, a maior profundidade foi a $25-30 \mathrm{~cm}$, com $75 \%$ do total a $12 \mathrm{~cm}$. O oxisol apresentava maior capacidade de retenção de água, menor densidade global, menor resistência ao penetrômetro, menor CTC, maior pH na camada de $20-40 \mathrm{~cm}$ e menor teor de alumínio trocável, em relação ao ultisol, que por sua vez apresentava compactação em todo o perfil.

BARRETO (1991) observou em um latossolo vermelho escuro que o sistema radicular do milho distribuiu-se em 78, 11 e $11 \%$ do total a 0 $20,20-40$ e $40-60 \mathrm{~cm}$ de profundidade, respectivamente, e o fato do horizonte $A_{3}(14-40 \mathrm{~cm})$ ter apresentado certa compactação provavelmente contribuiu para a maior presença de raízes a $0.20 \mathrm{~cm}$.

MANFRON et al. (1991) verificaram em uma terra roxa estruturada que, aos 71 dias após a semadura de milho, cerca de $70 \%$ do total da massa radicular até $1 \mathrm{~m}$ encontrava-se a $60 \mathrm{~cm}$ de profundidade. $O$ horizonte $B$ textural desse solo, presente até $105 \mathrm{~cm}$ de profundidade, onde ocorre a transição para o horizonte óxico, apresentava maiores teores de argila dispersa em água e de densidade global do solo, composta por blocos compactados de tamanho variável ou blocos angulares. Esses aspectos apresentam efeitos na geometria porosa do solo e seu maior reflexo apareceu até $60 \mathrm{~cm}$ de profundidade, onde apenas cerca de 6 a $7 \%$ dos diâmetros dos poros eram maiores que $0,05 \mathrm{~mm}$.

TAYLOR \& KLEPPER (1973) observaram que a condutividade hidráulica do solo teve um efeito muito maior na absorção de água pelo milho quando as densidades de raiz foram baixas; quando da presença de uma maior densidade radicular, seu efeito foi menor, pois as raízes são, em média, mais velhas e frequentemente menos permeáveis, sendo a maior parte da água extraída pelas jovens, que estão em uma proporção decrescente à densidades mais altas. As raízes mais profundas usualmente podem extrair mais água e de maneira mais efetiva do que aquelas próximas à superfície do solo, devido ao fato delas serem mais jovens, menos comprimidas e frequentemente localizadas em solo úmido.

MENGEL \& BARBER (1974) observaram que o fluxo de nutrientes foi maior para as plantas de milho mais jovens (20 dias), diminuindo até cerca de 70 dias, pois o aumento no comprimento das raízes foi mais rápido que o da taxa de absorção nesse período. A partir de 70 dias, o comprimento cresceu a uma menor taxa, aproximadamente igual à taxa de absorção de nutrientes, o que fez com que o fluxo desses elementos permanecesse praticamente uniforme, com cerca de $5 \%$ daquele ocorrido a 20 dias. Esse maior fluxo de nutrientes nas plantas jovens indica que um nível muito maior de disponibilidade de $\mathrm{N}$ no solo é necessário durante os primeiros estádios que nos demais.

EDWARDS \& BARBER (1976) mostraram que o comprimento das raízes de milho aumentou exponencialmente com o tempo e que o valor máximo do fluxo de $\mathrm{N}$ para as raízes ocorreu a 18 e 21 dias, com uma queda exponencial até 58 dias após a germinação, quando o fluxo correspondeu a cerca de $5 \%$ do maior valor.

Em condições de campo e em casa de vegetação, HATLITLIGIL et al. (1984) observaram que o hîbrido de milho que apresentou maior massa radicular teve uma maior produção de matéria seca total e de grãos. A capacidade de utilização de nutrientes, juntamente com a proliferação do sistema radicular no solo demonstraram ser os principais fatores para um alto potencial de produção.

MACKAY \& BARBER (1986) afirmam que o comprimento máximo de raízes pode variar entre diferentes genótipos de milho, em função da disponibilidade de $\mathrm{N}$, podendo tal característica explicar a capacidade de absorção adicional desse nutriente após o pendoamento e exibir uma grande resposta à adubação nitrogenada.

SHAVIV \& HAGIN (1990) observaram que a aplicação de fertilizante nitrogenado a $5-8 \mathrm{~cm}$ proporcionou uma maior densidade de raiz na 
camada superficial do solo a 46,58 e 75 dias após a semeadura, e uma maior produção de grãos de milho, em comparação a aplicação de $\mathrm{N}$ a $30-35$ $\mathrm{cm}$ de profundidade.

CRESTANA et al. (1991) apresentaram o potencial da técnica de processamento de imagens como ferramenta poderosa nos estudos da ciência do solo, principalmente na avaliação de raízes e na morfologia do solo. Em CRESTANA et al. (1994), foi apresentado o sistema SIARCS (Sitema Integrado para Análise de Raízes e Cobertura do Solo).

Assim sendo, o objetivo desse trabalho o de analisar a distribuição radicular do milho irrigado em uma terra roxa estruturada latossólica, em função da aplicação de $120 \mathrm{hg} / \mathrm{ha}$ de $\mathrm{N}$ via solo e via água de irrigação, através da técnica de processamento de imagens de raízes em um perfil do solo de $1 \mathrm{~m}$ de profundidade.

\section{MATERIAL E METODOS}

O experimento foi conduzido em uma terra roxa estruturada latossólica, de textura argilosa (TORRADO \& SPAROVEK, 1993). A TABELA 1 apresenta a análise química e a TABELA 2, a análise granulométrica e a densidade global do solo.

O preparo do solo constituiu-se de uma subsolagem, gradagem e aração em maio de 1992. A semeadura manual realizou-se em 9 de junho, em uma área total de $1000 \mathrm{~m}^{2}$, utilizando-se sementes de milho híbrido duplo AG-519, variedade essa adaptada ao cultivo de inverno (safrinha), com espaçamento entre linhas de $1 \mathrm{~m}$ e adubação de $30 \mathrm{~kg} / \mathrm{ha}$ de $\mathrm{P}_{2} \mathrm{OS}$, na forma de superfosfato simples, e de $20 \mathrm{~kg} / \mathrm{ha}$ de $\mathrm{K}_{2} \mathrm{O}$, na forma de cloreto de potássio, em conformidade com a análise química do solo. Em função da aplicação de 120 $\mathrm{kg} / \mathrm{ha}$ de $\mathrm{N}$ na forma de uran, foram estabelecidos dois tratamentos dentro dessa área: (I) irrigação + fertilização nitrogenada via solo, ao lado das linhas de plantas, aos 13 e 47 dias após a emergência (dae), com as respectivas doses de 40 e $80 \mathrm{~kg} / \mathrm{ha}$ de N; (F) fertirrigação nitrogenada aos $34,49,64$, 82 e 101 dae, nas doses de 4, 6, 45, 30 e $35 \mathrm{~kg} / \mathrm{ha}$ de $\mathrm{N}$, respectivamente. Cada tratamento foi composto por 12 linhas de plantas, com $10 \mathrm{~m}$ de comprimento e foram espaçados em $18 \mathrm{~m}$ para evitar que jatos de água de um atingissem o outro. Nas entre linhas de cada tratamento foram instaladas 4 baterias de tensiômetros a 10, 20,35, $50,65,75,85,100$ e $115 \mathrm{~cm}$ de profundidade e tubos de alumínio para o acesso da sonda de nêutrons para estimativa da umidade do solo a 25 , $50,75 \mathrm{e} 100 \mathrm{~cm}$ de profundidade. $O$ gradiente do potencial total da água no solo $(\Psi \mathrm{t})$, correspondente à soma do potencial matricial $(\Psi \mathrm{m})$ e gravitacional (z), foi definido como a diferença entre os potenciais acima e abaixo da profundidade referida, dividido pela distância entre ambos, representando fluxos descendentes quando positivos e ascendentes quando negativos.

A irrigação foi efetuada para elevar a umidade do solo na camada superficial a valores em torno da capacidade de campo ( $-70 \mathrm{~cm}$ de coluna de água), quando o potencial matricial da água no solo de pelo menos um tensiômetro atingisse $-500 \mathrm{~cm}$ de coluna de água a $20 \mathrm{~cm}$, no início do desenvolvimento da cultura (até a irrigação realizada aos 49 dae), e a $35 \mathrm{~cm}$ de profundidade a medida que o sistema radicular se desenvolveu (a partir da irrigação realizada aos 63 dae). A quantidade de água armazenada no solo a $25,50,75$ e $100 \mathrm{~cm}$ de profundidade foi obtida integrando-se os valores de $\theta$ ao longo da profundidade $\mathbf{z}$.

A injeção do fertilizante fluido uran foi feita através de uma bomba injetora de acionamento hidráulico, conectada à tubulação condutora de água ao tratamento fertirrigado. As épocas de fertilização foram baseadas na marcha de absorção de $\mathrm{N}$ pelas plantas de milho irrigado determinada por BASSOI (1990), juntamente com a necessidade de irrigação.

TABELA 1. Análise química do solo terra roxa estruturada latossólica.

\begin{tabular}{cccccccccc}
\hline \hline $\begin{array}{c}\text { Prof. } \\
\mathrm{cm}\end{array}$ & $\mathrm{pH}$ & $\mathrm{pH}$ & $\mathrm{M} . \mathrm{O}$. & $\mathrm{P}$ & $\mathrm{Ca}$ & $\mathrm{Mg}$ & $\mathrm{K}$ & $\mathrm{Al}+\mathrm{H}$ & $\mathrm{V}$ \\
\hline $0-20$ & 5,18 & $\mathrm{H} \mathrm{O}$ & $\%$ & $\mu \mathrm{g} / \mathrm{cm}^{3}$ & $\ldots \ldots \ldots \ldots . \mathrm{meq} / 100$ & $\mathrm{~cm}^{3} \ldots \ldots \ldots \ldots \ldots$ & $\%$ \\
\hline $20-40$ & 5,32 & 6,30 & 1,53 & 34 & 4,94 & 0,84 & 0,34 & 2,7 & 69 \\
\hline \hline
\end{tabular}


TABELA 2. Análise granulométrica e densidade global do solo terra roxa estruturada latossólica.

\begin{tabular}{cccccc}
\hline \hline prof. cm & $\begin{array}{c}\text { argila } \\
\%\end{array}$ & $\begin{array}{c}\text { silte } \\
\%\end{array}$ & $\begin{array}{c}\text { areia fina } \\
\%\end{array}$ & $\begin{array}{c}\text { areia grossa } \\
\%\end{array}$ & $\begin{array}{c}\text { dens. global } \\
\mathbf{g}^{\mathbf{3}} \mathbf{c m}^{\mathbf{3}}\end{array}$ \\
\hline $0-20$ & 36,8 & 31,9 & 24,4 & 6,9 & 1,41 \\
$20-35$ & 43,8 & 34,5 & 19,6 & 2,1 & 1,50 \\
$35-50$ & 43,1 & 38,8 & 13,9 & 4,2 & 1,54 \\
$50-65$ & 57,6 & 20,7 & 16,6 & 5,1 & 1,47 \\
$65-75$ & 54,5 & 19,8 & 19,5 & 6,2 & 1,48 \\
$75-85$ & 55,4 & 15,2 & 21,8 & 7,6 & 1,42 \\
$85-100$ & 53,2 & 19,2 & 20,6 & 7,0 & 1,27 \\
\hline \hline
\end{tabular}

A evapotranspiração máxima do milho (ETm) foi obtida com os dados de evaporação do tanque classe $A$ do posto meteorológico instalado ao lado da área experimental, e com os valores dos coeficientes de tanque e de cultura relatados por DOORENBOS \& PRUIT (1975).

Aos 126 dae analisou-se o 'sistema radicular do milho, através de uma trincheira aberta longitudinalmente ds linhas de plantas em cada um dos tratamentos. Após a retirada da camada adensada originada pelas ferramentas na abertura da trincheira e com o auxílio de um reticulado de $1 \times 1 \mathrm{~m}$, subdividido em 25 quadrados de $20 \times 20 \mathrm{~cm}$, efetuou-se a filmagem do perfil do solo e das raízes expostas de 5 plantas por metro linear. As imagens obtidas foram digitalizadas por uma placa digitalizadora para IBM-PC, com resolução espacial de 512 × 512 pixels e 256 tons de cinza, e analisadas através do sistema SIARCS, desenvolvido pelo Centro Nacional de Pesquisa e Desenvolvimento de Instrumentação Agropecuária, da Empresa Brasileira de Pesquisa Agropecuária (CNPDIA/ EMBRAPA), em São Carlos, SP. Na imagem de cada área de $20 \times 20 \mathrm{~cm}$ do reticulado, o sistema radicular foi diferenciado pelo contraste entre as cores das raízes (branco) e do solo (vermelho). Através de opções oferecidas pelo SIARCS, subdividiu-se cada área da imagem a ser analisada em quadrados de 0,5 x 0,5 cm e de $1,0 \times 1,0 \mathrm{~cm}$, num total de 1600 e 400 , respectivamente, de onde quantificou-se $o$ número destes que representavam as raízes, possibilitando assim a obtenção de sua densidade em cada área do reticulado.
Aos 150 dae, efetuou-se a colheita em 3 linhas de $10 \mathrm{~m}$ lineares por repetição em cada tratamento para a estimativa da produção de grãos.

\section{RESULTADOS E DISCUSSÃO}

Pela TABELA 3, pode-se observar que a densidade de raiz a $0-60 \mathrm{~cm}$ de profundidade foi maior em I, enquanto que a $60-100 \mathrm{~cm}$ de profundidade, o tratamento $F$ apresentou maiores valores. Em termos percentuais, nota-se que comparativamente os valores na camada de 0-20 cm são maiores em I, enquanto que nas demais, são superiores em $\mathrm{F}$. Alem disso, o sistema radicular no tratamento I apresentou uma redução mais acentuada entre as camadas de $40-60$ e de 60$80 \mathrm{~cm}$, em relação aos tratamento $F$. Tal fato pode ser em decorréncia da aplicação de toda a dose de N no tratamento I ate 47 dae, O que pode ter proporcionado essa maior presença de raízes na camada superficial, pois de acordo com MENGEL \& BARBER (1974) e EDWARDS \& BARBER (1976), o maior fluxo de $N$ para as raízes ocorre durante os primeiros estádios de desenvolvimento da cultura.

De maneira geral, a maior parte do sistema radicular concentrou-se ate a profundidade de $40 \mathrm{~cm}$ em ambos os tratamentos. Entre a duas opções de análise utilizadas $(0,5 \times 0,5 \mathrm{~cm}$ e $1,0 \times$ $1,0 \mathrm{~cm}$ ), os valores apresentaram uma variação de $5,8,6,4,12,1,7,8$ e $13,6 \%$ em I e de 2,2, 6,6, $9,8,5,2 \in 13,8 \% \mathrm{em} F$, para as camadas de 0-20, $20-40,40-60,60-80$ e 80-100 cm de profundidade, respectivamente. 
TABELA 3. Densidade (D) e distribuição percentual do sistema radicular do milho nos tratamentos irrigado (I) e fertirrigado (F) aos 126 dae.

\begin{tabular}{ccccccccc}
\hline \hline camada & D I & \% I & D F & $\%$ F & D I & $\%$ I & D F & $\%$ F \\
cm & $0,5 \times 0,5$ & $0,5 \times 0,5$ & $0,5 \times 0,5$ & $0,5 \times 0,5$ & $1,0 \times 1,0$ & $1,0 \times 1,0$ & $1,0 \times 1,0$ & $1,0 \times 1,0$ \\
\hline $0-20$ & 918 & 58,3 & 583 & 45,9 & 2235 & 61,9 & 1238 & 44,8 \\
$20-40$ & 372 & 23,6 & 327 & 25,7 & 797 & 22,1 & 662 & 24,0 \\
$40-60$ & 168 & 10,7 & 151 & 11,9 & 339 & 9,4 & 365 & 13,2 \\
$60-80$ & 81 & 5,2 & 138 & 10,9 & 171 & 4,7 & 317 & 11,5 \\
$80-100$ & 35 & 2,2 & 71 & 5,6 & 69 & 1,9 & 179 & 6,5 \\
total & 1574 & 100,0 & 1270 & 100,0 & 3611 & 100,0 & 2761 & 100,0 \\
\hline \hline
\end{tabular}

TABELA 4. Contribuição percentual das camadas de solo na variação do armazenamento de água no solo $(\Delta \mathrm{A})$ nos tratamentos irrigado (I) e fertirrigado $(\mathrm{F})$.

\begin{tabular}{|c|c|c|c|c|c|c|c|c|}
\hline $\begin{array}{c}\text { periodo } \\
\text { dae }\end{array}$ & $\begin{array}{c}\Delta \mathrm{A} \mathrm{I} \\
0-25 \mathrm{~cm}\end{array}$ & $\underset{25-50 \mathrm{~cm}}{\Delta A}$ & $\underset{50-75 \mathrm{~cm}}{\Delta A}$ & $\underset{75-100 \mathrm{~cm}}{\Delta \mathrm{A} \mathrm{I} \mathrm{I}^{\mathrm{I}}}$ & $\begin{array}{c}\Delta A F \\
0-25 \mathrm{~cm}\end{array}$ & $\underset{25-50 \mathrm{~cm}}{\Delta \mathbf{F}}$ & $\underset{50-75 \mathrm{~cm}}{\Delta A F}$ & $\underset{75-100 \mathrm{~cm}}{\Delta A F}$ \\
\hline $29-41$ & 51,20 & 31,65 & 6,68 & 10,47 & 43,25 & 30,09 & 14,48 & 12,22 \\
\hline $43-48$ & 33,97 & 31,84 & 11,82 & 22,37 & 39,14 & 33,22 & 18,63 & 9,01 \\
\hline $52-56$ & 33,08 & 31,03 & 24,36 & 11,53 & 44,33 & 29,56 & 15,27 & 10,84 \\
\hline $58-63$ & 25,30 & 28,18 & 27,12 & 19,40 & 19,93 & 31,18 & 32,54 & 16,35 \\
\hline $65-80$ & 41,25 & 31,93 & 17,58 & 9,24 & 41,99 & 32,29 & 16,97 & 8,75 \\
\hline $94-101$ & 40,39 & 31,07 & 17,16 & 11,38 & 38,78 & 32,12 & 20,38 & 8,72 \\
\hline
\end{tabular}

Em consequência da presença de raízes, a amplitude de variação do potencial matricial da água no solo foi maior a $10,20,35 \mathrm{e} 50 \mathrm{~cm}$ que a $65,75,85,100$ e $115 \mathrm{~cm}$ de profundidade (figuras 1 a 3), e a variação do armazenamento de água no solo recebeu maior contribuição de 0-25 e de 25-50 $\mathrm{cm}$ de profundidade (TABELA 4). Essas variações de armazenamento foram obtidas a partir de 29 dae, quando o sistema radicular já apresentava um certo desenvolvimento e em intervalos de tempo em que a quantidade de água armazenada no solo foi reduzida pela evapotranspiração e drenagem interna. $O$ gradiente do potencial total da água no solo (figuras 4 a 9) mostram que ocorreram valores negativos com maior frequência nas profundidades de $20,35,50$ e $65 \mathrm{~cm}$, que nas de 75, 85 e $100 \mathrm{~cm}$, em ambos os tratamentos.

Os valores de evapotranspiração máxima do milho durante o período de inverno, em geral, foram crescentes, apesar de que em alguns dias entre 65 e 103 dae, os mesmos apresentaram-se muito baixos. Entre 23 e 89 dae, a reposição de água ocorreu principalmente por meio de irrigação $(118,1 \mathrm{~mm})$, já que as precipitações ocorridas tota-

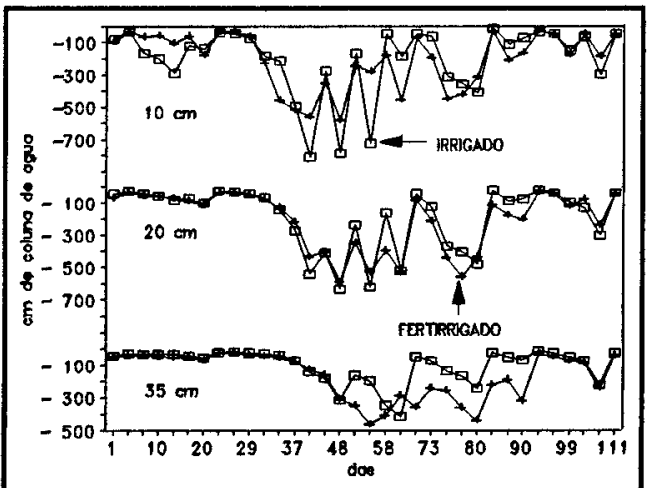

Figura 1. Potencial matricial médio da água no solo a 10,20 e $35 \mathrm{~cm}$ de profundidade.

lizaram apenas $26,8 \mathrm{~mm}$, enquanto a ETm acumulada nesse período foi de $167,0 \mathrm{~mm}$. Essas informações indicam que mesmo as camadas mais profundas do perfil do solo de $1 \mathrm{~m}$ podem ter contribuído para o processo de evapotranspiração, graças à distribuição do sistema radicular apresentada na TABELA 2. REICHARDT et al. (1979) verificaram para a cultura do milho em um 
latossolo vermelho amarelo, fase arenosa, a contribuição de camadas do solo de até cerca de $130 \mathrm{~cm}$ de profundidade no processo de evapotranspiração. ESPINOZA (1980) observou em um latossolo vermelho escuro de cerrado na fase de máximo desenvolvimento vegetativo do milho, uma extração relativa de água de $43,26,19$ e $12 \%$, para as camadas de 0-30, 30-60, 60-90 e 90-120 $\mathrm{cm}$ de profundidade, respectivamente.

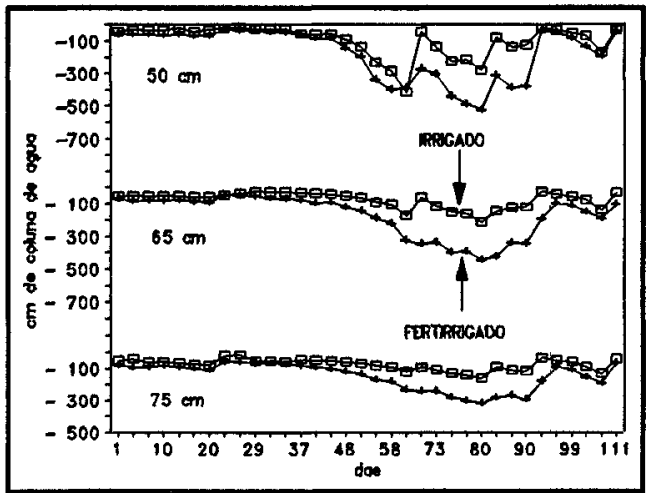

Figura 2 - Potencial matricial médio da água no solo a 50,65 e $75 \mathrm{~cm}$ de profundidade.

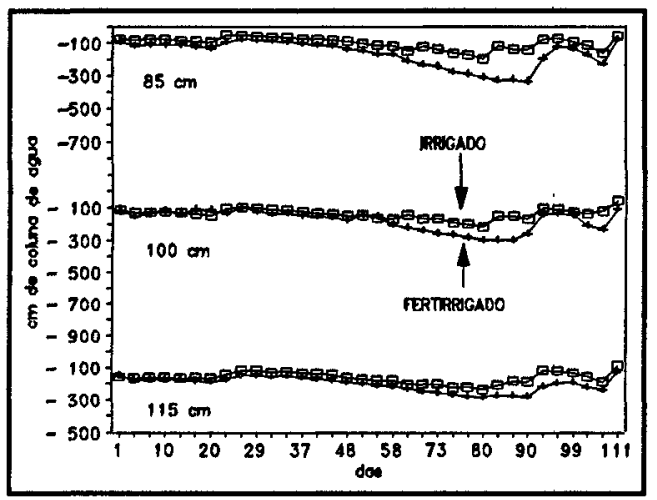

Figura 3 - Potencial matricial médio da água no solo a 85,100 e $115 \mathrm{~cm}$ de profundidade.

Essa contribuição das camadas mais profundas do solo no processo de extração de água pelas plantas fez com que gradientes negativos aparecessem mesmo a maiores profundidades (figuras 5,8 e 9). Os valores negativos a $75 \mathrm{~cm}$ no tratamento $F$ apresentaram-se maiores em módulo e durante um maior período de tempo em relação à I, e esse comportamento pode estar relacionado

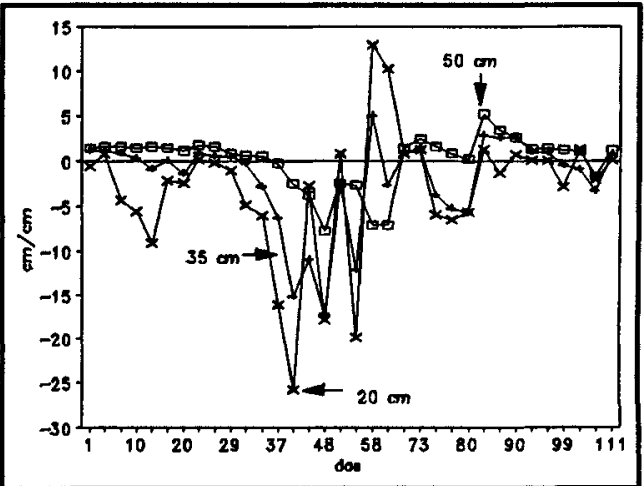

Figura 4 - Gradiente do potencial total médio da água no solo a 20,35 e $50 \mathrm{~cm}$ de profundidade no tratamento irrigado.

com a redução menos pronunciada da distribuição percentual das raízes, entre as camadas de 40-60 e de $60-80 \mathrm{~cm}$ de profundidade nesse tratamento (TABELA 2). Pelas Figuras 1 a 3 , onde observa-se uma maior diferença entre os valores de $\Psi \mathrm{m}$ em uma fase de grande desenvolvimento vegetativo (63 e 93 dae), indicando que a extração de água em F foi mais profunda.

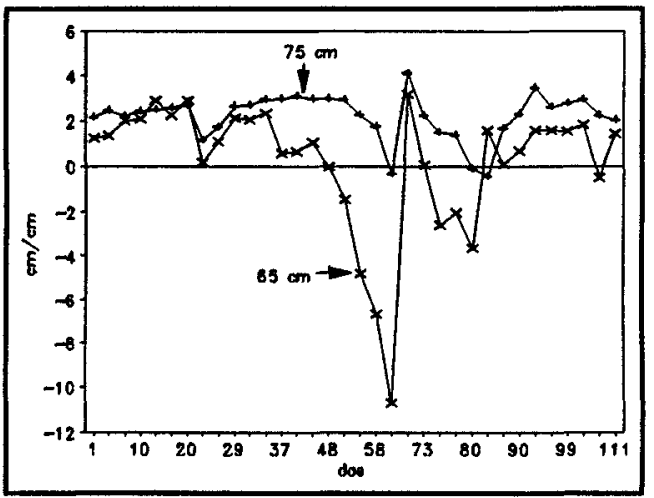

Figura 5 - Gradiente do potencial total médio da água no solo a 65 e $75 \mathrm{~cm}$ de profundidade no tratamento irrigado.

ESPINOZA (1980) verificou que as maiores diferenças quanto à extração de água em 4 densidades de semeadura de milho coincidiram com os maiores valores do coeficiente de cultura. Isso acarretaria em diferentes valores de ETm para cada tratamento, mas acredita-se que tais diferenças não seriam significativas para o cálculo de balanços hídricos. 


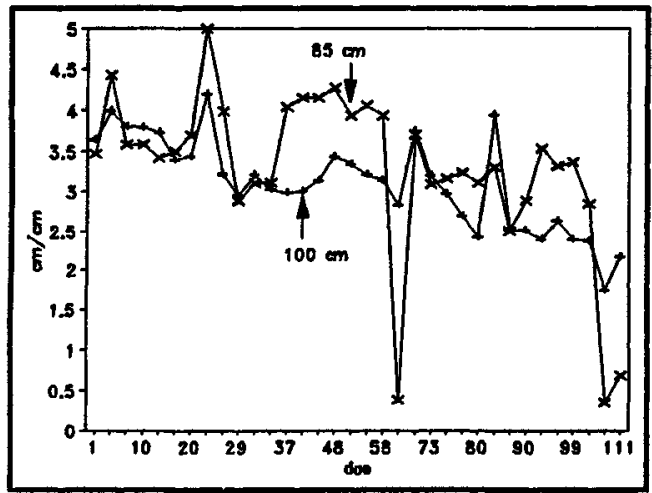

Figura 6 - Gradiente do potencial total médio da água no solo a 85 e $100 \mathrm{~cm}$ de profundidade no tratamento irrigado.

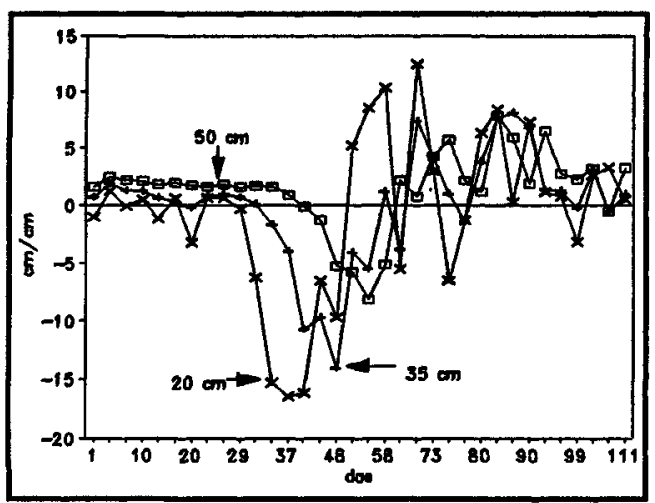

Figura 7 - Gradiente do potencial total médio da água no solo a 20,35 e $50 \mathrm{~cm}$ de profundidadeno tratamento fertirrigado.

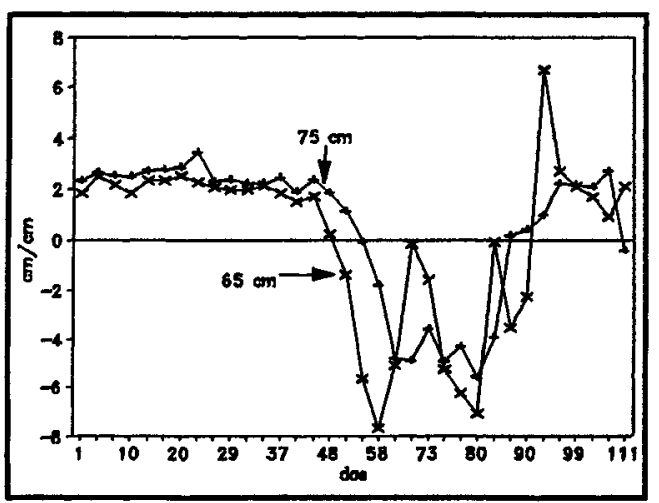

Figura 8 - Gradiente do potencial total médio da água no solo a 65 e $75 \mathrm{~cm}$ de profundidadeno tratamento fertirrigado.

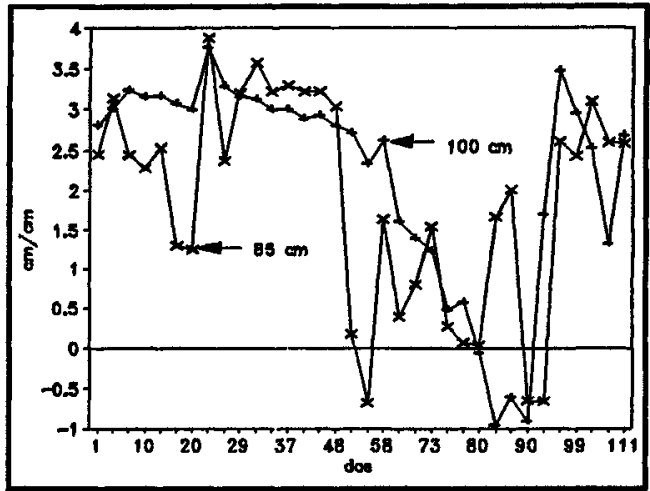

Figura 9 - Gradiente do potencial total médio da água no solo a 85 e $100 \mathrm{~cm}$ de profundidade no tratamento fertirrigado.

Apesar da maior densidade de raiz a 0-60 cm de profundidade em I, a produção de grãos nesse tratamento foi de $4516 \mathrm{~kg} / \mathrm{ha}$, inferior ao do tratamento $F$, de $5072 \mathrm{~kg} / \mathrm{ha}$, sendo que tais valores médios diferiram entre si pelo teste $t$ a $10 \%$ de probabilidade.

\section{CONCLUSÃO}

A densidade de raiz de milho a $0-60 \mathrm{~cm}$ de profundidade foi maior na aplicação de $\mathrm{N}$ via solo, enquanto que a $60-100 \mathrm{~cm}$ de profundidade a fertirrigação proporcionou uma maior presença de rázes. Em termos percentuais, a adubação via solo proporcionou uma maior distribuição apenas a 0 $20 \mathrm{~cm}$ de profundidade. Apesar disso, a maior produção de grãos de milho foi obtida com a fertirrigação.

De acordo com as análises do sistema radicular através do SIARCS, cerca de $70 \%$ das raízes concentraram-se a $0-40 \mathrm{~cm}$ de profundidade, independente do modo de aplicação de $N$. Os resultados de distribuição radicular obtidos através dessa técnica apresentaram uma boa correspondência com os obtidos pelos tensiómetros (variação do potencial matricial da água no solo), e sonda de nêutrons (variação do armazenamento de água no solo).

\section{REFERENCIAS BIBLIOGRAFTCAS}

BARRETO, A. C. Efeito do sistema de rotaçũo, sucessão - niveis de calagem sobre caracteristicas fisicas e químicas do solo e no desenvolvimento radicular e produção de gráos de mitho (Zea mays L.). 
Piracicaba, 1991. 154p. Dissertação (Mestrado) Escola Superior de Agricultura "Luiz de Queiroz", Universidade de Sao Paulo.

BASSOI, L.H. Lixiviação e acumulação de macronutrientes pelo milho (Zea mays L.) com e sem irrigação suplementar. Botucatu, 1990. 102p. Dissertação (Mestrado) - Faculdade de Ciências Agronómicas, Universidade Estadual Paulista.

CRESTANA, S.; GUMARĀES, M.F.; JORGE, L.A.C.; TOZZI, C.L.; TORRE, A.; VAZ, C.M.P.; RALISCH, R. Avaliação do crescimento de rázes e morfologia do solo auxiliada por processamento de imagens. In: CONGRESSO BRASILEIRO DE CIÊNCIA DO SOLO, 23., 1991, Porto Alegre. Resumos... Porto Alegre: Sociedade Brasileira de Ciência do Solo, 1991. p.130.

CRESTANA, S.; GUIMARÃES, M.F.; JORGE, L.A.C.; RALISCH, R.; TOZZI, C.L.; TORRE, A.; VAZ, C.M.P. Avaliação da distribuição de raízes no solo auxiliada por processamento de imagens digitais. Revista Brasileira de Ciencia do Solo, Campinas, 1993. (no prelo).

DEMATTE, J.L.I. Characteristics of Brazilian soils related to root growth.In:THE SOI/ROOT SYSTEM IN RELATION TO BRAZILIAN AGRICULTURE, 1980, Londrina. Proceedings... Londrina: Instituto Agronómico do Paraná, 1981. p.21-41.

DOORENBOS, J.; PRUIT, W.O. Guidelines for predicting crop water requirements. Rome: FAO, 1975. 179p.

EDWARDS, J.H.; BARBER, S.A. Nitrogen uptake characteristics of corn roots at low $\mathbf{N}$ concentration as influenced by plant age. Agronomy 'Journal, Madison, v.68, n.1, p.17-19, 1976.

ESPINOZA, W. Extração de Ggua pelo milho em latossolo da regiāo dos cerrados. Pesquisa Agropecúria Brasileira, Brasilia, v.15, n.1, p.69-78, 1980.

HATLITLIGL, M.B.; OLSON, R.A.; COMPTON, W.A. Yield, water use, and nutrient uptake of com hybrids under varied irrigation and nitrogen regimes. Fertilizers Research, The Hague, v.5, n.3, p.321333, 1984.

MACKAY, A.D.; BARBER, S.A. Effect of nitrogen on root growth of two corn genotypes in the field. Agronomy Journal, Madison, v.78, n.4, p.699-703, 1986.
MANFRON, P.A.; PAULETTO, E.A.; LIBARDI, P.L.; MORAES, S.O. Efeito do método de preparo do solo na distribuiç̧̃o radicular do milho (Zea mays L.) em terra roxa estruturada. Ciencia Rural, Santa Maria, v.21, n.3, p.353-366, 1991.

MENGEL, D.B.; BARBER, S.A. Rate of nutrient uptake per unit of corn root under field conditions. Agronomy Journal, Madison, v.66, n.3, p.399-402, 1974.

REICHARDT, K. Soil physico-chemical conditions and the development of roots. In: THE SOIL/ROOT SYSTEM IN RELATION TO BRAZILIAN AGRICULTURE, 1980, Londrina. Proceedings... Londrina: Instituto Agronómico do Paraná, 1981. p.103-114.

REICHARDT, K.; LIBARDI, P.L.; SAUNDERS, L.C.U.; CADMA Z.A. Dinâmica da água em solo cultivado com milho. Revista Brasileira de Ciencia do Solo, Campinas, v.3, n.1, p.1-5, 1979.

SHAVIV, A.; HAGN, J. Interaction of root distribution of com with fertilizer placement and frequency of irrigation in lysimeters. Fertilizers Research, The Hague, v.28, n.1, p.49-54, 1991.

TAYLOR, H.M.; KLEPPER, B. Rooting density and water extraction patterns of com (Zea mays L.). Agronomy Journal, Madison, v.65, n.6, p.965-968, 1973.

TORRADO, P.V.; SPAROVEK, G. Mapa geológico detalhado do Campus Luiz de Queiróz da Universidade de Sro Paulo. Piracicaba, Escola Superior de Agricultura Luiz de Queiróz, 1993. Escala 1:10000.

WAHAB, A.; TALLEYRAND, H.; LUGO-LOPEZ, M.A. Rooting depth, growth and yield of com as affected by soil water avaiability in an Utisol and an Oxisol. The Journal of the Agriculture of University of Puerto Rico, Puerto Rico, v.60, n.3, p.316-328, 1976.

Recebido para publicaçāo em 30.06 .94

Aceito para publicação em 26.08.94 